\title{
An enhanced particle swarm optimization algorithm
}

\author{
Wameedh Riyadh Abdul-Adheem \\ Department of Electrical Power Engineering Techniques, Al-Mamoun University College, Baghdad, Iraq
}

\begin{tabular}{l} 
Article Info \\
\hline Article history: \\
Received Feb 28, 2019 \\
Revised Mar 28, 2019 \\
Accepted Jun 25, 2019 \\
\hline Keywords: \\
Exponential decay inertia \\
weight \\
Particle swarm optimization \\
Stochastic optimization \\
algorithm
\end{tabular}

\begin{abstract}
In this paper, an enhanced stochastic optimization algorithm based on the basic Particle Swarm Optimization (PSO) algorithm is proposed. The basic PSO algorithm is built on the activities of the social feeding of some animals. Its parameters may influence the solution considerably. Moreover, it has a couple of weaknesses, for example, convergence speed and premature convergence. As a way out of the shortcomings of the basic PSO, several enhanced methods for updating the velocity such as Exponential Decay Inertia Weight (EDIW) are proposed in this work to construct an Enhanced PSO (EPSO) algorithm. The suggested algorithm is numerically simulated established on five benchmark functions with regards to the basic PSO approaches. The performance of the EPSO algorithm is analyzed and discussed based on the test results.
\end{abstract}

Copyright $\odot 2019$ Institute of Advanced Engineering and Science. All rights reserved.

\section{Corresponding Author:}

Wameedh Riyadh Abdul-Adheem, Department of Electrical Power Engineering Techniques, Al-Mamoun University College,

Baghdad, Iraq.

Email: wameedh.r.abduladheem@almamonuc.edu.iq

\section{INTRODUCTION}

In recent decades, search algorithms based on stochastic have been broadly exploited to find solutions for practical problems that are optimal. These algorithms have an opportunity of discovering the optimal global solution as compared to the gradient-based algorithms. The PSO is the modern stochastic algorithm. Initially, this algorithm was introduced in 1995 [1]. The general idea is to mimic the behavior of species such as fishes, birds or bees lookout for food. Exchanging information is required for making all the particles in a swarm move in the direction of single target stochastically and independently. Different paths are explored by distinct particles, which allow them to discover a wide range of searching space and extended the possibility of obtaining a solution, which is globally optimal. The main advantage of the PSO algorithm as compared to the Genetic Algorithm (GA) is that the programming of the PSO algorithm does not require any encoding /decoding procedures. The searching for a Global Optimal Solution (GOS) may affect by Incorrect selection of some of the PSO algorithm parameters [1].

The basic PSO algorithm experiences premature convergence due to the rapid dropping of diversity [2]. In addition, it suffers from a low speed of convergence, particularly on the complicated problem [3]. For these reasons, several modifications were previously considered to improve the performance of the basic PSO. In [2], a diversity enhancing procedure and search strategies in the neighborhood were engaged. In [4], the improved PSO was founded on back-Propagation neural network. In [3], an adaptive mutation was utilized. In [5], a segmentation PSO (SePSO) approach was proposed, in which the segmentation is utilized to obtain the global and local optimal solution. In [6], a Dynamic Objective Function Environment was purposed based on improved PSO to increase the capability to a quick reaction to the environment change. In [7], two enhanced PSO develops were propose, which founded on functional constriction factor and functional inertia weight. In [4], the basic PSO velocity formula was split into two parts and the two learning factors were upgraded. In [8], a mutation operator based on isotropic Gaussian is 
utilized to enhance the PSO algorithm. In [4], the theory of Evolutionary Game was utilized to improve the PSO algorithm.

In a field of engineering, the PSO algorithm was utilized extensively [9]. A load frequency control system based on PSO-fuzzy logic approach was proposed in [10]. A model identification of governor-turbine for a single power plant by using SePSO algorithm was proposed in [5]. Improved PSO was utilized in different techniques for image processing [11]. Finally, a survey of PSO algorithm applications in antenna circuit was reported in [12].

In this paper, the proposed PSO algorithm is constructed by allowing the parameters of the basic algorithm to be adopted depending on the iteration variable. Moreover, some of the particles' positions in a swarm are updated based on bounded random fashion. These modifications are suggested for the purpose that the particles diversity is increased in a swarm for increasing the opportunity of obtaining the GOS.

Endure of the paper is organized in the following: the PSO is a brief discussion in Section 2. The approach of the proposed EPSO will be designated in Section 3. The test functions, setting of the parameters and outcomes will be established in Section 4. Lastly, in Section 5 the conclusions are illustrated.

\section{THE BASIC PSO}

In basic PSO, a problem of optimization leans to obtain a set of parameter $x=\left(x_{1}, x_{2}, \ldots, x_{m}\right)^{t}$ of dimension $m$ variables i.e.

$$
\text { Minimize } f(x) \text { subject to } x_{i}^{L} \leq x_{i} \leq x_{i}^{U}, i=1,2, \ldots, m
$$

Algorithm 1 offerings the procedure of basic PSO. In PSO logarithm, each $i^{\text {th }}$ particle in a swarm of $n$ particles moving in a space of $d$-dimension is characterized by its position and velocity that are labeled as $X_{i}=\left(x_{i 1}, x_{i 2}, \ldots, x_{i d}\right)^{T}$ and $V_{i}=\left(v_{i 1}, v_{i 2}, \ldots, v_{i d}\right)^{T}$, respectively. In the original PSO algorithm, the update law of the particles' velocity and position are described as $[1,13]$.

$$
\begin{aligned}
& V_{i}^{t+1}=V_{i}^{t}+c_{1} r_{1}\left(x_{\text {pbest }}-X_{i}^{t}\right)+c_{2} r_{2}\left(x_{\text {gbest }}-X_{i}^{t}\right) \\
& X_{i}^{t+1}=X_{i}^{t}+V_{i}^{t+1}
\end{aligned}
$$

where $c_{1}$ is the cognitive acceleration coefficient, and $c_{2}$ is the social acceleration coefficient, $r_{1}$ and $r_{2}$ are the random values between $[0,1], x_{\text {pbest }}$ is the personal best of the particle and $x_{\text {gbest }}$ is the global best of the particle. $X_{i}^{t}$ is the current position of $i^{t h}$ particle at iterationa $t . V_{i}^{t}$ is the velocity of $i^{t h}$ particle at iteration $t$. Ma oreover, to prevent premature convergence an inertia weighting factor was added to (1). The modified updating law of the velocity (2) was suggested as [13].

$$
V_{i}^{t+1}=\omega V_{i}^{t}+c_{1} r_{1}\left(x_{\text {pbest }}-X_{i}^{t}\right)+c_{2} r_{2}\left(x_{\text {gbest }}-X_{i}^{t}\right)
$$

where $\omega$ is the inertia weight.

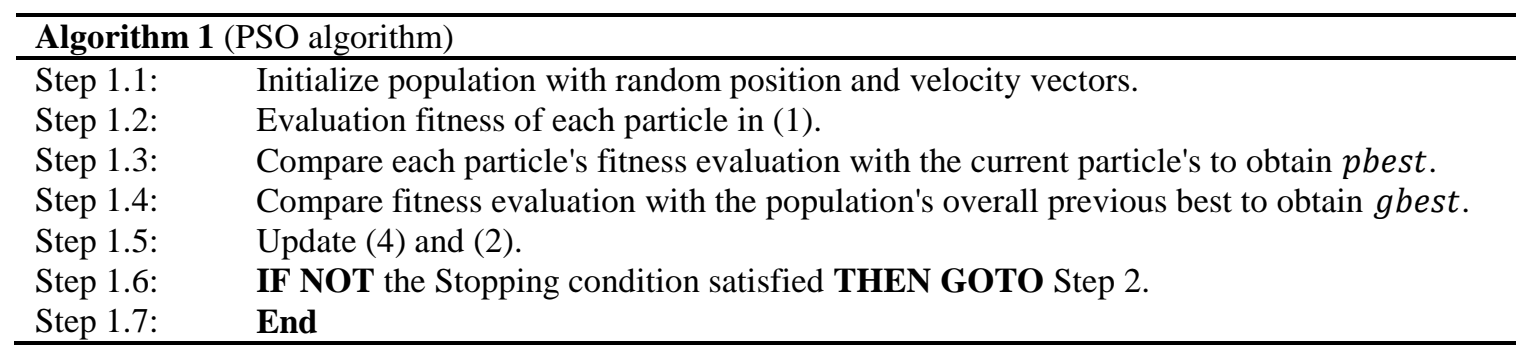

\section{ENHANCED PARTICLE SWARM OPTIMIZATION (EPSO)}

The proposed EPSO has been developed through two main steps. The first enhancement involves the adoption of the basic algorithm parameters $\omega, c_{1}$, and $c_{2}$ through the use of the iteration variable $t$ to determine the current values of the basic parameters. Modifying (4) to be expressed as:

$$
\omega^{t}=\omega_{0} \exp \left(-\mu_{\omega}{ }^{t} / t_{\max }\right)
$$




$$
\begin{aligned}
& c_{1}^{t}=c_{01}\left(1+\mu_{c 1} t / t_{\text {max }}\right) \\
& c_{2}^{t}=c_{02}\left(1+\mu_{c 2}{ }^{t} / t_{\text {max }}\right) \\
& V_{i}^{t+1}=\omega^{t} V_{i}^{t}+c_{1}^{t} r_{1}\left(x_{\text {pbest }}-X_{i}^{t}\right)+c_{2}^{t} r_{2}\left(x_{\text {gbest }}-X_{i}^{t}\right)
\end{aligned}
$$

where $\omega^{t}$ is the current EDIW, $c_{1}^{t}$ is the current cognitive acceleration coefficient , $c_{2}^{t}$ is the current social acceleration coefficient at iteration $t$, and $t_{\text {max }}$ is the largest time of iterations. The initial values of the parameters $\omega, c_{1}$, and $c_{2}$ are $\omega_{0}, c_{01}$, and $c_{02}$,respectively. This enhanced algorithm is implement in the aid of three parameters which are $\mu_{\omega}, \mu_{c 1}$, and $\mu_{c 2}$.

The second enhancement is implemented for the reason that the particles diversity is increased in a swarm for increasing the opportunity of result the GAS in which $X_{i}^{t}$ posthe ition of some of the particles in a swarm is modified randomly within a certain range according to:

$$
\begin{aligned}
& \text { If } r_{3}>P_{r 3} \text { THEN } X_{i}^{t+1}=X_{i}^{t}+V_{i}^{t+1}\left(1+2 P_{V}\left(r_{4}-0.5\right)\right) \text {, } \\
& \text { ELSE } X_{i}^{t+1}=X_{i}^{t}+V_{i}^{t+1}
\end{aligned}
$$

where $r_{3}$ and $r_{4}$ are the random values between $[0,1], P_{r 3}$ is the percentage of particles covered by velocity change and $P_{V}$ is the maximum percentage increase in speed the of particles.

\section{NUMERICAL SIMULATION}

For the purpose of testing the suggested enhanced algorithm, five test functions (Booth function $\left(f_{0}\right)$, Holder-Table function $\left(f_{1}\right)$, McCormick function $\left(f_{2}\right)$, McCormick function $\left(f_{2}\right)$, Sphere Function $\left(f_{3}\right)$, Three-Hump Camel Function $\left(f_{4}\right)$ ) are itemized in Table 1. For the purpose of relating the EPSO with basic PSO; all the five test functions are repetitive 1000 times. The Standard Deviation (STD) and Minimum Value (MIN) are selected to reveal the algorithms advantages and disadvantages. In this experiment, the parameters of the basic PSO and the EPSO are listed in Table 2. The numerical results of applying both algorithms of the five test functions are listed in Table 3 . The results indicated in Table 3 clearly illustrates the superiority of the proposed algorithm to the basic algorithm as a result of the change the value of the algorithm parameters based on time and randomly modifying the position of particles.

Table 1. The benchmark functions $[14,15]$

\begin{tabular}{lll}
\hline Definition & Search domain & The global minimum \\
\hline$f_{0}(x)=\left(x_{1}+2 x_{2}-7\right)^{2}+\left(2 x_{1}+x_{2}-5\right)^{2}$ & $x_{i} \in[-10,10]$, & $f\left(x^{*}\right)=0$, \\
& $i=1,2$. & $x^{*}=(1,3)$ \\
$f_{1}(x)=-\left|\sin \left(x_{1}\right) \cos \left(x_{2}\right) \exp \left(\left|1-\frac{\sqrt{\left\{x_{1}^{2}+y_{2}^{2}\right\}}}{\pi}\right|\right)\right|$ & $x_{i} \in[-10,10]$, & $f\left(x^{*}\right)=-19.2085$, \\
$f_{2}(x)=\sin \left(x_{1}+x_{2}\right)+\left(x_{1}-x_{2}\right)^{2}-1.5 x_{1}+2.5 x_{2}+1$ & $i=1,2$ & $x^{*}=( \pm 8.05502, \pm 9.66459)$ \\
& $x_{1} \in[-1.5,4]$, & $f\left(x^{*}\right)=-1.9133$, \\
$f_{3}(x)=x_{1}^{2}+x_{2}^{2}$ & $x_{i} \in[-10,10]$, & $x^{*}=(-0.54719,-1.54719)$ \\
& $i=1,2$. & $f\left(x^{*}\right)=0$, \\
$f_{4}(x)=2 x_{1}^{2}-1.05 x_{1}^{4}+\frac{x_{1}^{6}}{6}+x_{1} x_{2}+x_{2}^{2}$ & $x_{i} \in[-5,5]$, & $x^{*}=(0,0)$ \\
& $i=1,2$ & $f\left(x^{*}\right)=0$, \\
\end{tabular}

Table 2. The parameters of both the basic PSO and EPSO

\begin{tabular}{ccc}
\hline Algorithm & Parameter & Value \\
\hline \multirow{2}{*}{ PSO } & $\omega$ & 0.5 \\
& $c_{1}$ & 0.12 \\
& $c_{2}$ & 1.2 \\
& $\omega_{0}$ & 0.5 \\
& $\mu_{\omega}$ & 1 \\
\multirow{2}{*}{ EPSO } & $t_{\max }$ & 50 \\
& $c_{01}$ & 0.12 \\
& $\mu_{c 1}$ & 0.5 \\
& $c_{02}$ & 1.2 \\
& $\mu_{c 2}$ & 0.5 \\
\hline
\end{tabular}


Table 3. The Comparison results

\begin{tabular}{cccc}
\hline Function & Algorithm & STD & MIN \\
\hline \multirow{2}{*}{$f_{0}$} & PSO & 18.2114 & $2.5773 * 10^{-18}$ \\
& EPSO & 8.9607 & $2.4550 * 10^{-27}$ \\
$f_{1}$ & PSO & 4.9935 & -19.2085 \\
& EPSO & 2.4711 & -19.2085 \\
$f_{2}$ & PSO & 0.5522 & -1.9132 \\
& EPSO & 0.2120 & -1.9132 \\
$f_{3}$ & PSO & 2.8072 & $6.1199 * 10^{-19}$ \\
& EPSO & 1.0940 & $1.3916 * 10^{-28}$ \\
$f_{4}$ & PSO & 28.3077 & $5.2100 * 10^{-18}$ \\
& EPSO & 1.6891 & $2.2693 * 10^{-29}$ \\
\hline
\end{tabular}

\section{CONCLUSION}

The premature is one of the disadvantages of the basic PSO. This problem is solved by proposing the EPSO Algorithm. The formula of the velocity and position update are improved. The ability of the EPSO is validated through five benchmark functions and the results were good in function optimization.

\section{REFERENCES}

[1] T. Y. Chen and T. M. Chi, "On the improvements of the particle swarm optimization algorithm," Adv. Eng. Softw., vol. 41, pp. 229-239, 2010.

[2] H. Wang, et al., "Diversity enhanced particle swarm optimization with neighborhood search," Inf. Sci. (Ny)., vol. 223, pp. 119-135, 2013.

[3] D. C. Tran, et al., "A New Approach of Diversity Enhanced Particle Swarm Optimization with Neighborhood Search," pp. 143-144, 2014.

[4] Z. Zhou and B. Jiao, "Improvement of Particle Swarm Optimization," PIERS Online, vol. 5, pp. 261-264, 2009.

[5] A. S. Jaber, et al., "A new parameters identification of single area power system based LFC using Segmentation Particle Swarm Optimization (SePSO) algorithm," Asia-Pacific Power Energy Eng. Conf. APPEEC, 2013.

[6] Y. Lu, et al., "Improved particle swarm optimization algorithm and its application in text feature selection," Appl. Soft Comput. J., vol. 35, pp. 629-636, 2015.

[7] X. Yan, et al., "An Improved Particle Swarm Optimization Algorithm and Its Application,” vol. 10, pp. 316-324, 2013.

[8] D. Furman, et al., "Enhanced Particle Swarm Optimization Algorithm: Efficient Training of ReaxFF Reactive Force Fields," J. Chem. Theory Comput., vol. 14, pp. 3100-3112, 2018.

[9] M. R. AlRashidi and M. E. El-Hawary, "A survey of particle swarm optimization applications in electric power systems,” IEEE Trans. Evol. Comput., vol. 13, pp. 913-918, 2009.

[10] A. S. Jaber, et al., "Advance Two-Area Load Frequency Control Using Particle Swarm Optimization Scaled Fuzzy Logic," Adv. Mater. Res., vol. 622-623, pp. 80-85, 2012.

[11] R. Wang, "Research on Image Processing Based on Improved Particle Swarm Optimization," 2018 10th Int. Conf. Meas. Technol. Mechatronics Autom., pp. 538-540, 2018.

[12] Q. Pi and H. Ye, "Survey of particle swarm optimization algorithm and its applications in antenna circuit," 2015 IEEE Int. Conf. Commun. Probl. ICCP 2015, pp. 492-495, 2016.

[13] G. Yang, "A modified particle swarm optimizer algorithm," 2007 8th Int. Conf. Electron. Meas. Instruments, ICEMI, pp. 2675-2679, 2007.

[14] M. Elkhechafi, et al., "Firefly Algorithm for Supply Chain Optimization," Lobachevskii J. Math., vol. 39, pp. 355-367, 2018.

[15] S. G. De-Los-Cobos-Silva, et al., "An Efficient Algorithm for Unconstrained Optimization," Math. Probl. Eng., vol. 2015, 2015. 